\title{
Infectious Diseases Data Observatory (IDDO) Visceral Leishmaniasis Library of Clinical Therapeutic Studies: A Protocol for a Living Systematic Review of Clinical Studies
}

\section{sauman Singh-Phulgenda ( $\nabla$ sauman.singh@iddo.org )}

University of Oxford https://orcid.org/0000-0003-2892-3053

\section{Sumayyah Rashan}

University of Oxford

\section{Eli Harriss}

University of Oxford Bodleian Library: University of Oxford

\section{Prabin Dahal}

University of Oxford

\section{Caitlin Naylor}

University of Oxford

\section{Matthew Brack}

University of Oxford

Philippe Guerin

University of Oxford

\section{Brittany J Maguire}

University of Oxford

\section{Protocol}

Keywords: Infectious diseases data observatory (IDDO), Visceral Leishmaniasis library, clinical therapeutic studies, Preferred Reporting Items for Systematic-Reviews

Posted Date: December 29th, 2021

DOI: https://doi.org/10.21203/rs.3.rs-1159661/v1

License: (9) (1) This work is licensed under a Creative Commons Attribution 4.0 International License. Read Full License 


\section{Abstract}

Introduction: Visceral Leishmaniasis $(\mathrm{VL})$ is a vector-borne disease caused by protozoan parasites of the genus Leishmania. The disease is endemic in parts of South Asia, East Africa, South America and the Mediterranean region, with an estimated 50,000 to 90,000 cases occurring annually. A living systematic review of existing scientific literature is proposed to identify clinical drug efficacy studies against $V L$, conducted following the Preferred Reporting Items for Systematic-Reviews and Meta-Analyses (PRISMA) guidelines.

Methods and analysis: The proposed living systematic review builds on a previous systematic review first carried out in 2016, and the current protocol is designed to capture any published or registered VL clinical study from Nov-2021 onwards. The following databases will be searched by a medical librarian: PubMed, Ovid Embase, Scopus, Web of Science Core Collection, Cochrane Central Register of Controlled Trials, clinicaltrials.gov, WHO ICTRP, as well as IMEMR, IMSEAR, and LILACS from the WHO Global Index Medicus. The systematic review will consider both randomised and non-randomised interventional studies, including single-armed studies.

Ethics and dissemination: A database of eligible studies, including study characteristics, is openly available (https://www.iddo.org/tool/vl-surveyor) and will be continually updated every six months. All findings will be published in a peer reviewed journal.

PROSPERO registration number: CRD42021284622

\section{Introduction}

Visceral leishmaniasis (VL), also known as Kala-Azar, is a neglected disease caused by protozoan parasites of the genus Leishmania. The disease is endemic in parts of the Indian subcontinent, East Africa, South America and the Mediterranean region, with an estimated 50,000 to 90,000 cases occurring in 2019 [1].

If untreated, the disease is generally fatal within 2 years [2]. Historically, pentavalent antimony (PA) had been the mainstay drug for VL treatment in the South Asia $[3,4]$. Due to the emergence and evolution of drug-resistant parasites and the poor safety profile of PA at higher doses [5-7], single-dose Liposomal Amphotericin B (L-AmB) has been recommended as the first-line therapy in the Indian subcontinent since 2010 [8]. PA remains the first-line therapy in East Africa - mainly as a combination regimen with paromomycin as drug resistance has not been reported and single dose L-AmB was found to have lower efficacy in this region $[9,10]$. Such heterogeneity in therapeutic responses has been thought to arise due to regional differences in parasite sensitivity/susceptibility, differences in underlying patient population, and genetic variability in parasite populations, although to date the exact reasons remain unclear [11]. A consolidation of evidence from existing therapeutic studies can provide further insights into host, 
parasite and determinants of drug resistance or treatment failure. Some major challenges towards such evidence consolidation include: VL studies are generally small and sparse with a median sample size of 32 (range: 1 - 3,126) patients per treatment arm (See S1 Data in [12]), VL control programmes are severely underfunded [13], and the drug supply is substantially lower than the estimated case burden of VL in several endemic countires [13], and little is known on the therapeutic efficacy of the exisiting drug reimens in pregnant patients [14]. It is therefore important to establish a robust and reliable repository of historical studies that can allow pooling data across available clinical trials to maximise the research potential and consequently the impact.

The infectious diseases data observatory (IDDO) VL library of clinical studies is an open-access resource that provides a systematically assembled inventory of published therapeutic studies [15]. The IDDO VL library was first initated in 2016 by searching the published literature from 1980 until 2016. After revising the search strategies adopted in 2019 , the library has been been updated bi-annually and the current approach taken to maintain the IDDO library of clinical studies follows a living systematic review methodology. A living systematic methodology is a periodic and incremental addition to a systematic review which saves resources and time that would be required to plan and conduct a new systematic review from scratch, minimising duplication and inefficiency [16]. Constantly identifying and adding information from new studies to the existing pool thus ensures that any subsequent meta-analysis is based on a robust database. This easily and freely accessible repository allows the selection of studies for inclusion in meta-analyses based on factors such as drug class, geography, and a number of host and parasite characteristics.

After the completion of the search update, the complied database of eligible studies is made publicly available to the global research community through the VL-surveyor on the IDDO website - which serves as a global "knowledge commons"[16]. Such an inventory can provide a unique resource to the VL research community for a better understanding of historical data on drug safety and efficacy, for planning aggregate data meta-analysis, to assess the potential volume of data available for subsequent data re-use, to assess the feasibility of carrying out individual participant data meta-analysis (IPD-MA) on a specific research topic, and help designing better prospective clinical trials.

This prospective protocol describes the living systematic review methodology used by IDDO to achieve the overarching aim of maintaining the VL library of clinical studies. The research objectives are:

1) The primary objective of this systematic review is to index all the clinical studies describing the efficacy of antileishmanial therapies and summarise the landscape of the clinical studies

2)The secondary objective is to extract the number of patients who develop the following clinical outcomes following treatment of VL within the study follow-up period in the studies identified by the review: a) relapse, b) initial cure, c) Lost to follow-up, d) Post-Kala Azar Dermal Leishmaniasis (PKDL). 


\title{
Material And Methods
}

A systematic review of the existing scientific literature to identify VL drug efficacy clinical trials will be conducted following the Preferred Reporting Items for Systematic-Reviews and Meta-Analyses (PRISMA) guidelines [17]. This protocol has been designed following the PRISMA-P recommendations (supplemental file 3). This review builds on a previous systematic review that had identified clinical trials registered or published before January 2016 [15]. The original search strategies were revised in 2019 and the systematic library has been updated bi-annually since then. The current protocol is designed to describe the methodology used by IDDO for the future scheduled update of the VL clinical study library (next search is scheduled for May 2022).

\section{Eligibility criteria}

All clinical studies in humans assessing any efficacy or safety outcome measure of antileishmanial drugs will be eligible for inclusion in this review. No restriction is placed on interventions/comparators used, outcomes measures adopted, or language of publication. Table 1 provides the description of the Population, Intervention, Comparison, Outcomes and Study design (PICOS) used for the systematic review.

Table 1

PICOS used for defining the search and eligibile studies

\begin{abstract}
PICOS Search String Strategy
Population - All clinical studies of Visceral Leishmaniasis in humans assessing any efficacy or safety outcomes of a antileishmanial drug.

- Studies on cutaneous leishmaniasis, post kala-azar dermal leishmaniasis (PKDL), canine $\mathrm{VL}$, and those evaluating vaccines or prophylactic usage of drugs, studies describing vector control, net distributions, prevalence estimation, and diagnostic tests will be excluded.
\end{abstract}

Intervention - The search strategy will be restricted to identify studies evaluating efficacy of antileishmanial interventions; non-interventional studies are therefore not considered.

Comparator - The search strategy and eligibility for inclusion is not restricted by the comparator used.

Outcomes - The search strategy and eligibility for inclusion is not restricted by treatment outcomes given the primary aim is to to understand the homogeneity/heterogeneity of all outcomes measured in VL.

- The following efficacy and safety outcomes of interest will be extracted from the studies identified: initial cure, relapse, lost to follow-up, post kala azar dermal leishmaniasis, death, adverse events and serious adverse events.

Study

Design
- Non-intervention studies, case reports, retrospective studies and individual studies enrolling fewer than six patients will be excluded. 


\section{Search strategy}

The following databases will be searched: PubMed, Ovid Embase, Scopus, Web of Science Core Collection, Cochrane Central Register of Controlled Trials, clinicaltrials.gov, WHO ICTRP, as well as IMEMR, IMSEAR, and LILACS from the WHO Global Index Medicus. The search will be run by an experienced librarian at the Bodleian Health Care Libraries, the University of Oxford every six month to capture new studies registered or published during the period. Thesaurus headings for Visceral Leishmaniasis will be used where available in combination with phrase searches (OR) constructed for the title or abstract fields ("black fever", "kala azar", "visceral leishmaniasis"). These search terms will be combined with filters for randomised controlled trials for PubMed (the box 3.a Cochrane Highly Sensitive Search Strategy)[18], Ovid Embase (an adapted version of the Box 3.e Cochrane Highly Sensitive Search Strategy) [18], with adaptations of both filters applied to Scopus and the Web of Science Core Collection. The VL search terms will also be combined with thesaurus and free text (title or abstract fields) searches for antileishmanial drugs including Pentamidine, Ambisome, Amphotericin. A search filter will be applied to exclude animal studies, and searches will be limited to the relevant publication dates for that search period. Complete details on the search terminologies used for different databases are presented in supplemental file 1.

\section{Study selection}

Two reviewers will screen the studies identified in the search against the pre-specified eligibility criteria (Table 1) in a blinded fashion using Covidence software for systematic reviews [19]. Disagreements will be resolved through discussion between the two reviewers, where possible, or alternatively, will be resolved by contacting a blinded third reviewer. Further details on the PICOS framework utilised for developing the search strategies and eligibility criteria for article screening is presented in supplemental file 1.

\section{Data extraction}

Pre-existing variable and data dictionaries with extraction rules developed in the earlier iteration of the systematic review will be used, and data extraction will be conducted in the REDCap electronic data capture tool hosted at the University of Oxford [20]. Data on the following aspects of the studies eligible for inclusion will be extracted: study status, publication year, study design, location, case definition, diagnostic method, parasite species, age and number of participants, inclusion and exclusion criteria, including the eligibility for pregnant women, malnourished individuals and comorbidities such as HIV and tuberculosis. Information will also be extracted regarding the treatments assessed for each arm, dosing regimen, number of patients cured, number of relapses, duration of follow-up and the number of patients who completed follow-up. Furthermore, the extraction will also capture safety parameters like the presence of an adverse events monitoring and classification system, informed consent, Data Safety and Monitoring Board and reported number of adverse events and serious adverse events including number of patients who died during the treatment or follow-up period. Lastly, parameters that would allow assessing the methodological rigour of studies such as information regarding sample-size estimation, 
randomisation method, allocation concealment and blinding of regimen or outcome assessment methodology will also be extracted. Full details of the variables extracted are presented in supplemental file 2.

\section{Meta-analysis}

A meta-analysis is planned for estimating the proportion of patients who develop relapse. This will be carried out using meta-analysis of single proportion using logit transformation. Heterogenity will be quantified using $R$ statistics and sub-group analyses will be carried by geographical regions, drug regimens and other study characteristics. Sensitivity analysis will be carried out by the adjudicated risk of bias status in the studies included in the specific meta-analysis. Further meta-analysis may be carried out in the future on the following safety and efficacy endpoints of interest: the proportion of patients who reach initial cure status, who are lost to follow-up and those who develop post kala azar dermal leishmaniasis (PKDL) and on the following safety endpoints: death, adverse events.

\section{Risk of bias assessment}

The primary objective of this systematic review is to index all the clinical studies describing the efficacy of antileishmanial therapies and summarise the landscape of the clinical studies including the chracteristics of the studies included and inclusion and exclusion criteria adopted. The analysis of this endpoint will be limited to providing a descriptive summary of trial characteristics, study design and interventions studied. Accordingly, assessment of meta-biases or the strength of the body of evidence represented within and across individual included records are not relevant for this research question. However, the biases related to the identification and inclusion of literature in this review and totality of evidence presented will be qualitatively discussed and assessed using the GRADE approach [21].

For a formal meta-analysis that will be conducted on the safety and efficacy endpoints of interest such as the number of patients with subsequent relapse, initial cure, lost to follow-up and those who develop post kala azar dermal leishmaniasis (PKDL) or death, risk of biases in the studies included will be conducted using the Cochrane risk of bias tools. Non-randomised comparative studies will be assessed using ROBINS-I tool and randomised studies will be asssed using Risk of bias 2 (RoB 2) tool [22, 23]. Single arm cohort studies will be asssed using Newcastle-Ottawa scale [24].

\section{Dissemination}

The compiled database of eligible studies after completion of six-monthly search will continue to be made publicly available through the IDDO webpage [25].

\section{Discussion}

The limited generation and availability of clinical trial data for poverty-related neglected tropical diseases like VL necessitates that systematic reviews be updated regularly to capture emerging evidence regarding drug safety, efficacy and resistance described in the latest studies. Such practice is even more important in the case of VL as most of the trials are small (the median sample-size of a clinical trial is 85 ), and the 
number of failures in any single study is smaller [15]. Constantly identifying and adding information from new studies to the existing pool is, therefore, crucial for the generation of robust evidence and to avoid duplication of work. Maintaining a living systematic review as proposed in this protocol will update the existing database periodically, thus providing a unqiue resource that will continuously update the evidence base. This publicly available resource is intended to support the wider VL research community and save resources and time that would otherwise be required by groups planning to conduct new systematic reviews from scratch [16]. Furthermore, availability of easily accessible data from past studies can act as basline for comparing estimates from new studies and for the data collected by national control programs in the field.

The IDDO VL library of clinical studies is publicly available under the Open Database License (ODbL) after completion of each round of review. This approach taken by IDDO serves as a global "knowledge commons", as the easily and freely accessible repository can inform further meta-analyses based on factors such as drug class, geography, and a number of host and parasite characteristics. Maintaining this initiative will continue to help facilitate up to date evidence that can assist researchers and policymakers alike in combating this disease. The utility of previous iterations of this database has already been demonstrated being used by the VL research community to understand different aspects of the disease including assessment of safety following antileishmanial therapies, characterisation of heterogeneity in patient population enrolled and in the design and conduct of VL clinical studies [12, 15, $26,27]$.

The initial goal of the IDDO VL library was to assess the feasibility of establishing an IPD platform to serve the research community. This goal is currently being realised as the 2017 review facilitated developing a VL data platform that currently hosts over 15,000 IPD from several studies identified from the review [28]. The VL research community has come together to draft research proposals to harness the scientific potential of the IPD platform in exploring different risk factors of therapeutic and safety outcomes on a large scale that otherwise would not be possible through the aggregate data metaanalysis [29].

The current living systematic review, therefore, not only serves as an active and comprehensive inventory of clinical studies in VL but also provides critical information to the VL community to identify research questions that would be feasible to answer using IPD meta-analysis. The promotion of these research activities remains critical as endemic countries are currently in different phases of disease control and elimination.

\section{Declarations}

\section{Authors' contributions}

Conceptualization : SSP, PD, PJG, BJM

Data Curation : N/A 
Formal Analysis : N/A

Funding Acquisition : PJG

Investigation : N/A

Methodology : EH, BJM

Project Administration : SSP, MB, BJM, PJG

Resources : PJG

Software : N/A

Supervision : PJG

Validation : SSP, SR, EH, MB, BJM

Visualization : N/A

Writing - Original Draft Preparation: SSP, PD, MB, BJM, PJG

Writing - Review \& Editing: SSP, SR, EH, PD, MB, CN, PJG, BJM

\section{Availability of data and material}

N/A

\section{Ethics approval and consent to participate}

Not applicable

\section{Consent for publication}

Not applicable

\section{Financial Disclosure Statement}

The review is funded by a Wellcome biomedical grant to the Infectious Diseases Data Observatory (Recipient: PJG; ref: 208378/Z/17/Z). The funders had no role in the design and analysis of the research or the decision to publish the work. 


\section{Competing interests}

None

\section{References}

1. WHO. Leishmaniasis. Key facts. In: WHO [Internet]. 2020 [cited 5 Jul 2020]. Available: https://www.who.int/news-room/fact-sheets/detail/leishmaniasis.

2. Burza S, Croft SL, Boelaert M, Leishmaniasis. Lancet. 2018;392:951-70. doi:10.1016/S01406736(18)31204-2.

3. Guerin PJ, Olliaro P, Sundar S, Boelaert M, Croft SL, Desjeux P, et al. Visceral leishmaniasis: current status of control, diagnosis, and treatment, and a proposed research and development agenda. Lancet Infect Dis. 2002;2:494-501. doi:https://doi.org/10.1016/S1473-3099(02)00347-X.

4. Croft SL, Olliaro P. Leishmaniasis chemotherapy-challenges and opportunities. Clin Microbiol Infect. 2011;17:1478-83. doi:10.1111/j.1469-0691.2011.03630.x.

5. Chulay JD, Spencer HC, Mugambi M. Electrocardiographic changes during treatment of Leishmaniasis with pentavalent antimony (sodium stibogluconate). Am J Trop Med Hyg. 1985;34:702-9. doi:10.4269/ajtmh.1985.34.702.

6. Thakur C. Harmful effect of high stibogluconate treatment of kala-azar in India. Trans R Soc Trop Med Hyg. 1986;80:672-3.

7. Ortega-Carnicer M, Alcazar R, De la Torre M, Benezet J. Pentavalent Antimonial-induced Torsade de Pointes. J Electrocardiotogy. 1997;30:143-5.

8. Singh OP, Singh B, Chakravarty J, Sundar S. Current challenges in treatment options for visceral leishmaniasis in India: A public health perspective. Infect Dis Poverty. 2016;5:1-15. doi:10.1186/s40249-016-0112-2.

9. Alves F, Bilbe G, Blesson S, Goyal V, Monnerat S, Mowbray C, et al. Recent Development of Visceral Leishmaniasis Treatments: Successes, Pitfalls, and Perspectives. Clin Microbiol Rev. 2018. doi:10.1128/CMR.00048-18.

10. Sundar S, Chakravarty J. An Update on Pharmacotherapy for Leishmaniasis. Expert Opin Pharmacother. 2015;16:237-52. doi:10.1517/14656566.2015.973850.

11. Khalil EAG, Weldegebreal T, Younis BM, Omollo R, Musa AM, Hailu W, et al. Safety and efficacy of single dose versus multiple doses of AmBisome for treatment of Visceral Leishmaniasis in Eastern Africa: A Randomised Trial. PLoS Negl Trop Dis. 2014;8:e2613. doi:10.1371/journal.pntd.0002613.

12. Singh-Phulgenda S, Dahal P, Ngu R, Maguire BJ, Hawryszkiewycz A, Rashan S, et al. Serious adverse events and mortality following treatment of Visceral Leishmaniasis: A systematic review and metaanalysis. PLoS Negl Trop Dis. 2021;15: e0009302. doi:https://doi.org/10.1371/journal. pntd.0009302. 
13. Choi HL, Jain S, Ruiz Postigo JA, Borisch B, Dagne DA. The global procurement landscape of leishmaniasis medicines. PLoS Negl Trop Dis. 2021;15:1-12. doi:10.1371/journal.pntd.0009181.

14. Dahal P, Singh-Phulgenda S, Maguire BJ, Harriss E, Ritmeijer K, Alves F, et al. Visceral Leishmaniasis in pregnancy and vertical transmission: A systematic literature review on the therapeutic orphans. PLoS Negl Trop Dis. 2021;15:e0009650. doi:10.1371/journal.pntd.0009650.

15. Bush JT, Wasunna M, Alves F, Alvar J, Olliaro PL, Otieno M, et al. Systematic review of clinical trials assessing the therapeutic efficacy of visceral leishmaniasis treatments: A first step to assess the feasibility of establishing an individual patient data sharing platform. PLoS Negl Trop Dis. 2017;11:1-16. doi:10.1371/journal.pntd.0005781.

16. Maguire BJ, Guérin PJ. A living systematic review protocol for COVID-19 clinical trial registrations. Wellcome Open Res. 2020;5:1-9. doi:10.12688/wellcomeopenres.15821.1.

17. Moher D, Liberati A, Tetzlaff J, Altman DG. The PRISMA Group (2009) Preferred Reporting Items for Systematic Reviews and Meta- Analyses: The PRISMA Statement. PLoS Med. 2009;6: e1000097. doi:10.1136/bmj.b2535.

18. Lefebvre C, Glanville J, Briscoe S, Littlewood A, Marshall C, Metzendorf M-I, et al. Technical supplement to Chapter 4: Searching for and selecting studies. In: Higgins JPT, Thomas J, Chandler J, Cumpston M, Li T, Page MJ, Welch VA, editors Cochrane Handbook for Systematic Reviews of Interventions Version 62. 2021. Available: https://training.cochrane.org/handbook/version6.2/chapter-4-tech-suppl.

19. Veritas Health Innovation. Covidence systematic review software. Melbourne, Australia.: www.covidence.org; 2019. Available: www.covidence.org.

20. Harris PA, Taylor DP, Thielke R, Payne R, Gonzalez J. N, et al. Research Electronic Data Capture (REDCap) - A metadata-driven methodology and workflow process for providing translational research informatics support. J Biomed Inf. 2009;42:377-81. doi:10.1016/j.jbi.2008.08.010.

21. The Grading of Recommendations Assessment. Development and Evaluation. [cited 17 Mar 2021]. Available: https://www.gradeworkinggroup.org/.

22. Sterne JAC, Savović J, Page MJ, Elbers RG, Blencowe NS, Boutron I, et al. RoB 2: A revised tool for assessing risk of bias in randomised trials. BMJ. 2019;366:1-8. doi:10.1136/bmj.14898.

23. Sterne JA, Hernán MA, Reeves BC, Savović J, Berkman ND, Viswanathan M, et al. ROBINS-I: A tool for assessing risk of bias in non-randomised studies of interventions. BMJ. 2016;355:1-7. doi:10.1136/bmj.i4919.

24. Wells G, Shea B, O'Connell D, Peterson J, Welch V, Losos M, et al. The Newcastle-Ottawa Scale (NOS) for assessing the quality of nonrandomised studies in meta-analyses. In: Ottawa Hospital Research Institute [Internet]. 2021 [cited 1 Dec 2021]. Available: http://www.ohri.ca/programs/clinical_epidemiology/oxford.asp.

25. Infectious Diseases Data Observatory. VL Surveyor. [cited 17 Mar 2021]. Available: https://www.iddo.org/tool/vl-surveyor. 
26. Dahal P, Singh-Phulgenda S, Olliaro LP, Guerin PJ. Gender disparity in patients enrolled in clinical trials of VL: a systematic review and meta-analysis. PLoS Negl Trop Dis. 2021;15: e0009204. doi:https://doi.org/10.1371/ journal.pntd.0009204.

27. Infectious Diseases Data Observatory. VL Research Activities. [cited 17 Mar 2021]. Available: https://www.iddo.org/vl/research/vl-research-activities.

28. IDDO VL Data Platform. Accessing data. [cited 17 Mar 2021]. Available: https://www.iddo.org/vl/data-sharing/accessing-data.

29. Infectious Diseases Data Observatory. VL Study Groups. 2021 [cited 17 Sep 2021]. Available: https://www.iddo.org/vl/research/vl-study-groups.

\section{Supplementary Files}

This is a list of supplementary files associated with this preprint. Click to download.

- Supplementaryfile110Dec2021.docx

- Supplementaryfile2.xIsx

- Supplementaryfile3PRISMAPchecklist10Dec2021.doc 